\title{
Photometry of the dust nova V705 Cassiopeiae
}

\author{
L. Hric ${ }^{1}$, K. Petrík ${ }^{2}$, Z. Urban ${ }^{1}$, and D. Hanžl ${ }^{3}$ \\ 1 Astronomical Institute of the Slovak Academy of Sciences, 05960 Tatranská Lomnica, The Slovak Republic \\ e-mail: hric@ta3.sk \\ 2 Department of Mathematics, Informatics and Physics, Pedagogical Faculty, University of Trnava, 91843 Trnava, \\ The Slovak Republic \\ e-mail: astropet@ta3.sk \\ 3 N. Copernicus Observatory and Planetarium, 61600 Brno, The Czech Republic
}

Received April 9; accepted June 10, 1998

\begin{abstract}
We present the results of our $U B V$ photoelectric photometry of V705 Cas (Nova Cassiopeiae 1993) obtained at the Skalnaté Pleso, Stará Lesná, Hlohovec and Brno Observatories. The observations cover the light curve development of this nova over its stages of dust formation and disappearance of the envelope. The results of the light curve analysis are presented. We have also used the visual brightness estimates of V705 Cas by the members of the VSNET network as an auxiliary data set in order to determine the nova's brightness behaviour as accurately as possible. The basic parameters of the brightness decline of the nova $t_{2, B}=40 \mathrm{~d}, t_{2, V}=33 \mathrm{~d}, t_{3, B}=63 \mathrm{~d}$, $t_{3, V}=61 \mathrm{~d}$, with the accuracy not worse than one day, were obtained on the basis of our light curves. The relevant value of $M_{B}=(-7.27 \pm 0.3) \mathrm{mag}$ and the value $M_{V}=(-7.32 \pm 0.22) \mathrm{mag}$ in the maximum of brightness were derived. The corresponding value of the mass of the white dwarf component of the presumable underlying nova binary is $M_{\mathrm{WD}}=(0.79 \pm 0.06) M_{\odot}$. The colour excess at brightness maximum is $E_{B-V}=0.56 \mathrm{mag}$ and the corresponding value of the distance $r=(1.67 \pm 0.34) \mathrm{kpc}$. This value of the distance is very probably underestimated due to the strong extinction by the dust envelope of the nova. The general problem of the use of statistical calibrations as well as that of the distance determination for dust novae is discussed.
\end{abstract}

Key words: stars: novae, cataclysmic variables — stars: individual: V705 Cas

\section{Introduction}

Nova Cassiopeiae 1993, which later received the denotation V705 Cas, was discovered by Japanese amateur as-

Send offprint requests to: L. Hric tronomer K. Kanatsu on Dec. 7, 1993 as an object not far beyond the naked-eye visibility threshold (of about 6.5 photographic magnitude on the discovery day), at the following coordinates: $\alpha=23^{\mathrm{h}} 39^{\mathrm{m}} 22.3^{\mathrm{s}}, \delta=+57^{\circ} 14^{\prime} 22.55^{\prime \prime}$, eq. 1950.0 (Kanatsu 1993). The nova has reached the brightness maximum ten days after its discovery on Dec. 17, 1993, i.e., on JD $=2449339.29$, with maximal values of the magnitude in the $V$ and $B$ colours of $\left(V_{\max }=\right.$ $5.53 \mathrm{mag}$ and $B_{\max }=6.14 \mathrm{mag}$, respectively (Munari et al. 1994b). Its progenitor has been identified on the POSS as a $B \approx 18 \mathrm{mag}$ star (Skiff 1993). The further development of the nova's light curve morphology has clearly demonstrated it belongs to the DQ Her subclass of novae.

The first optical spectrogram of this nova was obtained by Schmidt (1993), already four days before the maximum. The nova appeared to be a Fe-II class object (Williams 1992), with an emission spectrum including many Fe II, O I, Ca II as well as $\mathrm{H}_{\alpha}-\mathrm{H}_{\gamma}$ lines. Woodward \& Greenhouse (1993) reported the spectrum obtained on Dec. 15 with FWHM from Balmer lines of $1680 \mathrm{~km}^{-1}$. Shore et al. (1994b) concluded from the IUE observations that the optical luminosity of this nova was larger than its UV flux by about a factor of 10 .

The resemblance of V705 Cas to nova DQ Her was further strengthened by its spectral behaviour because its spectra obtained soon after the outburst maximum showed optically thick Fe II lines (Starrfield et al. 1995). These authors took the UV spectra of the nova (using the IUE satellite) just a few hours after the announcement of the discovery. Comparing these early spectra with a later one obtained on Jan. 28, 1994, they showed that the effective temperature of the shell of the nova had increased from $8000 \mathrm{~K}$ (on Dec. 13 1993) to $24000 \mathrm{~K}$ until the day they obtained the later spectrum, whereas its expansion velocity had decreased from $2000 \mathrm{~km}^{-1}$ to $1300 \mathrm{~km}^{-1}$ over the same interval. The identical results were presented by Hauschildt et al. (1994), who concluded that the radiation 
pressure alone was sufficient to attain the observed high expansion velocity of the shell. Besides that, on the basis of the interstellar lines of the doublet $\mathrm{Na}$ I D a Mg II, they have estimated the distance to the nova to be between 3 and $6 \mathrm{kpc}$ - which is, as they noted, inconsistent with the distance value (about $2 \mathrm{kpc}$ ) determined from the light curve. On the other hand, the comparison of the nova's properties with those of DQ Her yields the lower limit for the distance determination of $3.4 \mathrm{kpc}$.

Elkin (1995) presented the results of spectroscopic observations of V705 Cas with the 6-m telescope at the SAO. The spectrum of the nova exhibited a large number of emission and absorption features and two easily discernible radial velocity systems. The primary absorption spectrum displayed radial velocity of $-550 \mathrm{~km}^{-1}$ and the diffuse-enhanced one that of $-1330 \mathrm{~km}^{-1}$. Gehrz et al. (1995) have published infrared spectrophotometric and photometric observations of the dust shell of V705 Cas in the interval covering days 130 to 418 after the outburst. Their measurements suggest that the dust shell was optically thick at least until 131 days after the outburst, later it exhibited features of an optically thin shell composed of silicon grains and of some hydrocarbons. On Jul. 22, 1995, the expanding shell of the nova was also detected in the radio part of the spectrum by Eyres et al. (1996), while they were observing with the MERLIN array at the wavelength of $6 \mathrm{~cm}$. The angular dimension of the shell was then approximately 0.2 arcsec, which corresponded to the distance of $2.5 \mathrm{kpc}$. Shore et al. (1994a) have studied the composition of the dust shell. On the basis of the extinction curve, they concluded that the nature of the dust formed in the vicinity of the nova is strictly different from that of the typical interstellar material. Their observations can be interpreted by the existence of large silicon grains within the dust shell, assuming the simultaneous presence of the stellar wind.

Evans et al. (1996a,b) obtained the IR spectroscopic data for this nova and detected conspicuous bands of $\mathrm{CO}$ which have been present already during its brightness maximum. To our knowledge, the most recent paper published on V705 Cas presents the results of the IR spectroscopy obtained on day 367 after the maximum (Lynch et al. 1997). These authors mention the strong IR excess and deduce the shell temperature to be within the interval from $710 \mathrm{~K}$ to $320 \mathrm{~K}$, depending on the specific wavelength.

The light behaviour of V705 Cas in the prenova stage was investigated by Munari et al. (1994a) on the archival photographic plates of the Asiago Astrophysical Observatory collection. No significant brightness fluctuations of the progenitor were found. Hauschildt et al. (1994) have described the morphological features of the light curve of V705 Cas and pointed out its close resemblance to DQ Her. Both novae exhibited premaximum halt, then rapid increase to maximum, an eruption of several magnitudes shortly after the beginning of the maxi- mum plateau stage and slow early decline $=$ by $2-3$ mag over 2-3 months before they entered the deep decline of the transition stage. Retter \& Leibowitz (1995) performed CCD photometric observations of V705 Cas in the $I$ colour during 10 nights in Aug. and Sep. 1995. The period analysis of these data yielded the detection of light variations of this nova with the most probable period of 0.228 days and the amplitude of 0.05 mag. Boyle (1994), along with his collaborators, has analyzed the direct CCD images of the nova shell and detected an asymmetric emission shell with a radius of about 9 arcsec in the vicinity of the nova, which by far exceeds any reasonably expected dimensions of the presently ejected shell, given its observed expansion velocity. Therefore, he interprets the detected structure as a relic of the previous outburst of the nova which could have occurred some time between 1920 and 1960 .

Several authors have attempted to deduce the basic physical parameters of this nova preferentially on the basis of its brightness decline. Chochol et al. (1995) determined the parameters of the nova from the photometric observations obtained during the first 2 months which have elapsed after the light maximum. Their derived distance value was $2.36 \mathrm{kpc}$. Elkin (1995) has deduced the nova's distance using several independent methods. As for polarimetric observations, he presented the value of $940 \mathrm{pc}$. From the interstellar Ca II K line, he derived the distance of 880 pc. Finally, on the basis of light curve analysis, he attained the value of 1350 pc. Lynch et al. (1997) have determined the distance to the nova to be $1270 \mathrm{pc}$ on the basis of the visual observations obtained by the AAVSO members and of those communicated via VSNET, with a probable error of $\pm 370 \mathrm{pc}$.

\section{Observations}

The photoelectric observational material discussed in this paper was obtained in the interval between December 17, 1993 and December 1, 1994 at the Skalnaté Pleso (SP) and Stará Lesná (SL) Observatories of the Astronomical Institute of the Slovak Academy of Sciences (Cassegrain 600/7500 telescope equipped with the singlechannel photometer/digital converter system), at the Hlohovec Observatory (H) (Cassegrain 600/7500, singlechannel photometer/mechanical signal recording device) and at the Nicolaus Copernicus Observatory in Brno (B) (Nasmyth 400, single-channel photometer/digital converter). The moments of the individual observations, $U, B$ and $V$ magnitudes and colour indexes $(U-B)$ and $(B-V)$ are listed in Table 1 . The accuracy of the photometric observations is expressed by the error which did not exceed the following values (in the individual colours): $U \pm 0.03$, $B \pm 0.02$ and $V \pm 0.02$, all in mags.

The $V$ colour light curve constructed from the data listed in Table 1, incorporating also data from Munari et al. (1994b) and from the VSNET network, is depicted 


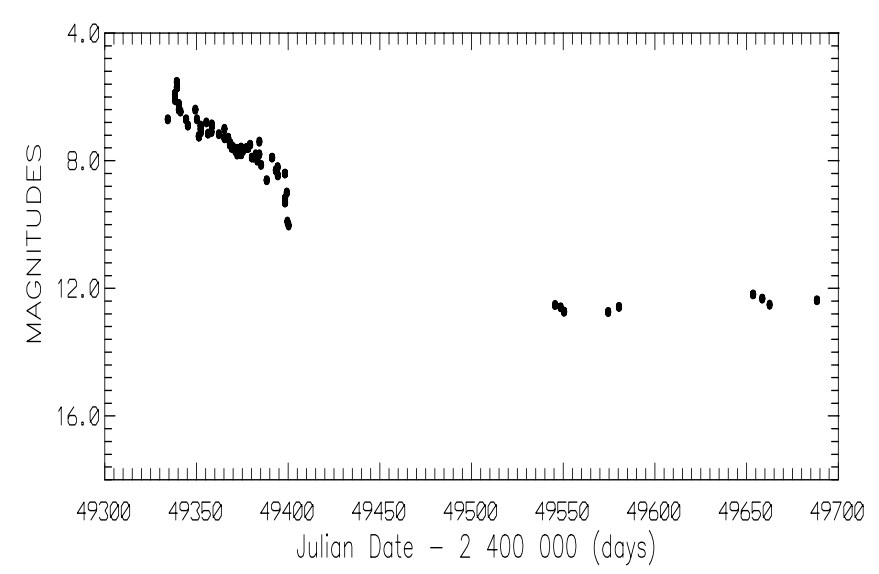

Fig. 1. Photoelectric observations of V705 Cas in the $V$ colour

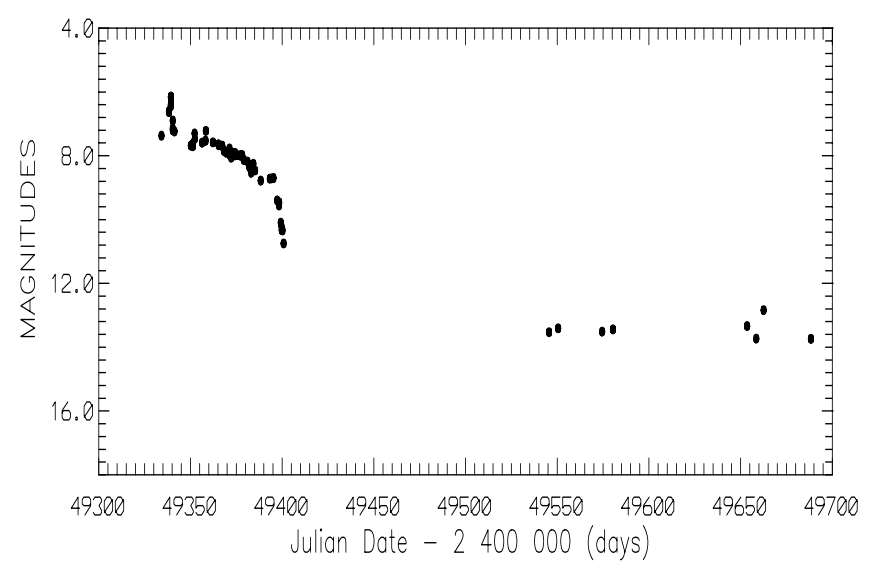

Fig. 2. Photoelectric observations of V705 Cas in the $B$ colour

in Fig. 1 and the $B$ colour one in Fig. 2. Both light curves are fitted by the polynome of the fourth degree, in order to determine $t_{3, V}$ and $t_{3, B}$ parameters accurately. We have selected the degree of the polynome from among several possibilities tested on the basis of the accuracy of the fitting of the observational data. The reason for the incorporation of the visual brightness estimates communicated via the VSNET network was to achieve a more complete and detailed description of the photometric behaviour of the nova. The light curve based solely on these visual data is displayed in Fig. 3.

\section{Results}

The principal morphological features of the light curve of V705 Cas can be described well on the basis of long-term visual brightness estimates displayed in Fig. 3. These data represent a quasi-regular coverage of an observational interval of more than 1000 days following the outburst. The light curve is characterized by a moderately rapid decline from the maximum brightness which is interrupted after approximately 50 days by a very abrupt decline to a minimum at the level of about $16.1 \mathrm{mag}$ which occurred on day
Table 1. Photoelectric observations of nova Cas 93

\begin{tabular}{rrrrrl}
\hline \hline & JDhel & & & & \\
Date & $244 . \ldots$ & $V$ & $U-B$ & $B-V$ & Obs. \\
\hline Dec. 17, 93 & 49339.377 & 5.68 & -0.07 & 0.56 & $\mathrm{~B}$ \\
29,93 & 49351.328 & 7.25 & -0.61 & 0.37 & $\mathrm{SP}$ \\
30, 93 & 49352.336 & 7.11 & -0.63 & 0.36 & $\mathrm{SP}$ \\
Jan. 03, 94 & 49356.334 & 7.15 & 0.38 & -0.56 & $\mathrm{~B}$ \\
05,94 & 49358.202 & 7.10 & -0.67 & 0.43 & $\mathrm{~B}$ \\
05,94 & 49358.283 & 6.86 & - & - & $\mathrm{H}$ \\
05,94 & 49358.380 & 6.90 & -0.51 & 0.32 & $\mathrm{SP}$ \\
09,94 & 49362.244 & 7.17 & -0.68 & 0.41 & $\mathrm{~B}$ \\
12,94 & 49365.262 & 7.27 & -0.71 & 0.37 & $\mathrm{~B}$ \\
12,94 & 49365.337 & 7.30 & -0.73 & 0.37 & $\mathrm{SP}$ \\
14,94 & 49367.287 & 7.28 & -0.76 & 0.4 & $\mathrm{~B}$ \\
15,94 & 49368.348 & 7.49 & -0.75 & 0.34 & $\mathrm{SP}$ \\
16,94 & 49369.410 & 7.54 & -0.77 & 0.36 & $\mathrm{SP}$ \\
18,94 & 49371.217 & 7.62 & -0.79 & 0.34 & $\mathrm{~B}$ \\
19,94 & 49372.228 & 7.68 & -0.77 & 0.34 & $\mathrm{~B}$ \\
19,94 & 49372.396 & 7.63 & -0.74 & 0.37 & $\mathrm{SP}$ \\
19,94 & 49372.416 & 7.63 & -0.67 & 0.34 & $\mathrm{SL}$ \\
21,94 & 49374.218 & 7.66 & -0.79 & 0.33 & $\mathrm{~B}$ \\
21,94 & 49374.333 & 7.59 & -0.72 & 0.32 & $\mathrm{SP}$ \\
22,94 & 49375.371 & 7.66 & -0.72 & 0.32 & $\mathrm{SP}$ \\
Feb. 01, 94 & 49385.270 & 8.13 & -0.79 & 0.33 & $\mathrm{H}$ \\
10,94 & 49394.342 & 8.46 & -0.57 & 0.25 & $\mathrm{SP}$ \\
14,94 & 49398.327 & 9.18 & -0.37 & 0.3 & $\mathrm{H}$ \\
14,94 & 49398.345 & 9.31 & -0.40 & 0.15 & $\mathrm{H}$ \\
16,94 & 49399.582 & 9.91 & -0.27 & 0.29 & $\mathrm{SL}$ \\
16,94 & 49400.269 & 10.03 & - & 0.31 & $\mathrm{~B}$ \\
Jul. 12,94 & 49545.549 & 12.52 & - & 1.01 & $\mathrm{H}$ \\
15,94 & 49548.567 & 12.59 & - & - & $\mathrm{H}$ \\
17,94 & 49550.519 & 12.73 & - & 0.68 & $\mathrm{H}$ \\
Aug. 09, 94 & 49574.475 & 12.74 & - & 0.77 & $\mathrm{H}$ \\
15,94 & 49580.414 & 12.58 & - & 0.86 & $\mathrm{H}$ \\
Oct. 28, 94 & 49653.538 & 12.19 & 0.3 & 1.15 & $\mathrm{H}$ \\
Nov. 02, 94 & 49658.548 & 12.32 & - & 1.41 & $\mathrm{H}$ \\
06,94 & 49662.574 & 12.51 & -0.28 & 0.33 & $\mathrm{H}$ \\
\hline \hline & & & & & \\
\hline & 49688.352 & 12.37 & 0.36 & 1.37 & $\mathrm{H}$ \\
\hline
\end{tabular}

103 after the maximum. Since then the brightness started to increase again and it can be assumed that by day 165 after the outburst the phase of the formation and development of the dust envelope had already been completed. The brightness of the nova on the shorter time scales appeared to be quite stable afterwards, exhibiting only relatively minor fluctuations of 0.5 mag at most. Nevertheless, the overall brightness of V705 Cas started to decline again soon. The nova has become fainter by $1.8 \mathrm{mag}$ (it declined slowly from $12.2 \mathrm{mag}$ to $14.0 \mathrm{mag}$ ) during about 1000 days which have elapsed since the formation and development of the dust shell were completed.

In order to study the phase of formation and development of the dust shell in more detail, we have analyzed the temporal change of the value of the $(U-B)$ index (Fig. 4). The variation of the index since the brightness maximum is characterized by a sharp decrease which can be associated with the heating of the nova's expanding shell. 


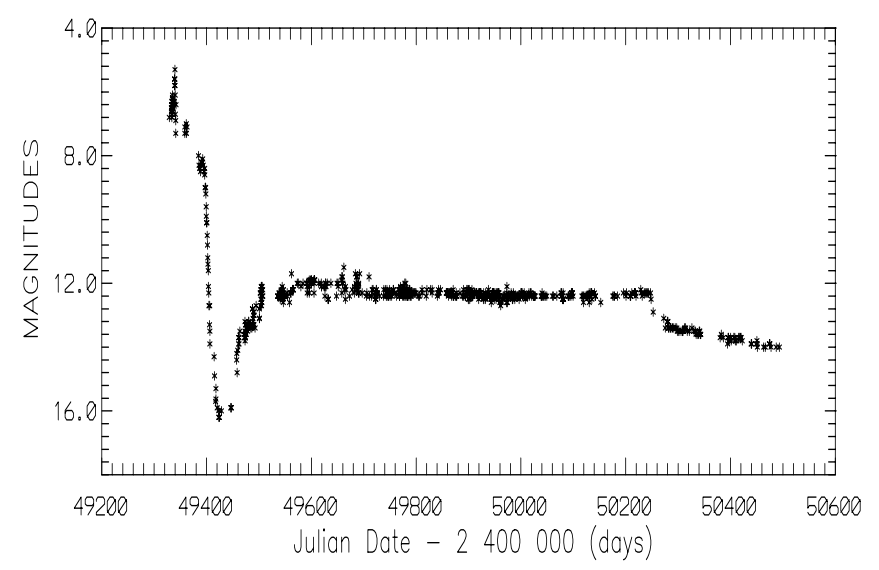

Fig. 3. Visual observations of V705 Cas

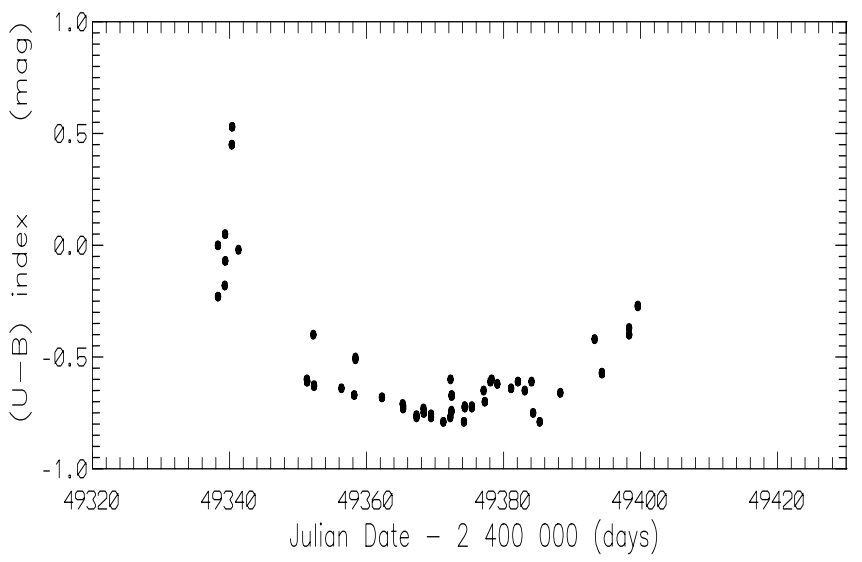

Fig. 4. The course of the development of the $(U-B)$ index during the early decline of V705 Cas

On day 33 after the maximum, the value of the index has become erratic. Then, on day 47 after the maximum, it started to exhibit an unambiguous sharp increase. Such a behaviour can be attributed to the cooling of the expanding shell and especially to the formation of the dust component of the shell. This interpretation is consistent with the behaviour of the $U B V$ photoelectric light curves of the nova which commonly exhibited an abrupt decline in all three colours at about 50 days after the maximum.

We have used the photoelectric data to determine the basic physical parameters of the nova. At first, we eliminated the data spanning the formation and development of the dust shell from the photoelectric light curves during the data processing. Then, we fitted the remaining data with polynomials of the fourth degree, in order to be able to determine the overall trend of the brightness decline more accurately. Thereby we obtained the following values: $t_{2, B}=40 \mathrm{~d}, t_{2, V}=33 \mathrm{~d}, t_{3, B}=63 \mathrm{~d}$, $t_{3, V}=61 \mathrm{~d}$, with the error of \pm 1 day. We have calculated the absolute magnitude of the nova at maximum in the $B$ colour according to the empirical relations derived by Pfau \& Livio (1992). This has provided us with the relevant values of $M_{B}=(-7.43 \pm 0.20) \mathrm{mag}$ and $M_{B}=(-7.27 \pm 0.20) \mathrm{mag}$, respectively. As for the $V$ colour, we have used the relation published by Schmidt (1957) and obtained the value $M_{V}=(-7.29 \pm 0.22) \mathrm{mag}$, as well as that by Della Valle \& Livio (1995) and obtained the value $M_{V}=(-7.34 \pm 0.20)$ mag. In order to be able to advance further and select the best value of the $M_{B}$, one needs to perform a short discussion of the methods used. Livio (1996) has compared the individual statistical calibrations for the determination of the absolute brightness of a nova at maximum and showed that the linear relation proposed by Pfau (1976) is fitting the data file worse than more modern Livio's relation. On this basis, we have decided to use in further calculations the value $M_{B}=(-7.27 \pm 0.20) \mathrm{mag}$ as the mean one and the value determined according to Pfau (1976) as the lower limit for the distance determination of the nova. Nevertheless, we realize that in order to be as exact as possible one should limit the application of these methods only to the use of fits by Della Valle \& Livio (1995) and Livio (1996) in the future.

For our further calculations, we have used $M_{V}=$ $(-7.32 \pm 0.22) \mathrm{mag}$, representing the mean of two values of this parameter presented above. The relation by Livio (1992) has enabled us to calculate the mass of the white dwarf component of the nova system responsible for the outburst of V705 Cas. We have obtained $M_{\mathrm{WD}}=$ $(0.79 \pm 0.06) M_{\odot}$. This mass of the white dwarf component then yielded the corresponding Eddington luminosity $M_{\text {Edd }}=(-6.49 \pm 0.06)$ mag. Using the bolometric correction B.C. $=-0.28,($ Livio 1994), we derived the Eddington luminosity in the $V$ colour, $M_{V \text {,Edd }}=(-6.21 \pm 0.06)$ mag. Comparing this value with the empirically determined value of $M_{V}=(-7.32 \pm 0.22)$ mag. In fact, Starrfield (1988) published the value of Eddington limit for slow novae as $M_{\mathrm{bol}}=-7 \mathrm{mag}$, on the other hands the models by Kato \& Hachisu (1994) and by Kato (1994) reduce the Eddington luminosity at corresponding value of $M_{V \text {,Edd }}=-6$ mag. Thus we can state that the outburst of nova V705 Cas was probably a super-Eddington one.

Using $V_{\max }=5.53 \mathrm{mag}$ and $B_{\max }=6.14 \mathrm{mag}$ (Munari et al. 1994b) and the intrinsic $(B-V)$ index (0.05 mag), the colour excess at brightness maximum for intermediate values $E_{B-V}=0.56 \mathrm{mag}$ and the corresponding visual extinction $A_{v}=1.74 \mathrm{mag}$. Using then the well-known distance modulus relation, the distance to V705 Cas $r=$ $1.67 \pm 0.34 \mathrm{kpc}$. We have deduced the error of the distance determination as follows: the extinction determined on the basis of the value of $M_{B}=(-7.43 \pm 0.20)$ mag according to Pfau (1976) is $A_{v}=2.23 \mathrm{mag}$ and the corresponding distance is $r=1.33 \mathrm{kpc}$. We consider this value to be the lower limit of the distance to the nova and the difference between it and the mean distance to be the value of the error. Within the upper limit of the distance values interval created in such a way, there is the value determined through the method according to Miroshnichenko (1988). 
We consider this value to represent the upper limit of the distance determination (see Discussion).

\section{Discussion}

Such a value for the distance to V705 Cas $(r=1.67 \pm$ $0.34 \mathrm{kpc}$ ) is higher than the relevant values presented by Elkin (1995) and Lynch et al. (1997). In contrast, it is substantially lower than those derived by Hauschildt et al. (1994) on the basis of the UV spectroscopic observations with the IUE satellite and by Eyres et al. (1996) from radio observations. The method of the distance determination to a nova through the measurement of the equivalent widths of the Ca II $\mathrm{K}$ interstellar line is handicaped by the sensitivity of this particular line to the contamination by the circumstellar lines (Chochol et al. 1997). Thus, we suggest to view the distance determination of V705 Cas resulting from this approach (880 pc; Elkin 1995) as underestimated. For better understanding of the contamination by the circumstellar dust envelope, we give the following suggestion. Observed Ca II K interstellar line is created by two sources of absorption. One is connected to the dust envelope around the nova and the second one is produced by the interstellar medium (IM) itself. Resulting equivalent width of such profile doesn't reflect the true distance to the nova. Moreover the composite profile is produced not only by the absorption in the IM but by the emission from the dust envelope as well.

On the other hand, the CCD observations have revealed that V705 Cas is surrounded by an older shell (or shells), too (Boyle 1994). This shell is substantially larger than the shell ejected during the 1993 outburst. Therefore, it is disputable whether the direct radio images show only the latter shell. This fact indicates that the star may have undergone multiple explosions with mass ejection in the past. It is highly probable that the expanding envelope ejected by the nova in 1993 has been fragmented due to its collision with the material still residing in the immediate circumsystem vicinity as the relic of the previous outburst(s). For this reason, one can assume that the angular diameter of the outer border of the latter shell observed by Eyres et al. (1996) is smaller than it would be if the collision of the two structures would have not occurred. As a direct consequence of this, the distance to the nova should be lower than that value of $2.5 \mathrm{kpc}$ which has resulted from radio observations. Thus, we suggest to consider this kind of distance determination as overestimated. Moreover, during the same outburst the ejection of matter could be characterized by different episodes of mass ejection. This yield higher unaccuracy in the distance determination from the radio method.

It should be noted here that the distance determination to a nova strongly depends on the adoption of the correct amount of the interstellar extinction. However, the relevant value could be overestimated just due to
Table 2. Review of the dependence of distance on various adopted values of colour excess

\begin{tabular}{lll}
\hline \hline$E_{B-V}$ & Distance & Reference \\
\hline 0.38 & 2.36 & Chochol et al. (1997) \\
0.43 & 2.01 & Elkin (1995) \\
0.5 & 2 & Hauschildt et al. (1994) \\
0.56 & 1.67 & this paper \\
0.67 & 0.94 & Elkin (1995) \\
1.09 & 1.27 & Lynch et al. (1997) \\
\hline \hline
\end{tabular}

the continuing presence of the relic dust shell around the nova at the time of its new outburst. This is also probably the case for V705 Cas. Therefore, the distance of $1.67 \mathrm{kpc}$ can be underestimated in part. For this reason, we have carried out the extinction determination more extensively, using several methods. We have obtained the lowest value of the colour excess $E_{B-V}=0.38$ as the result of the intercomparison of the colour indexes of the stars surrounding the nova selected from the SAO catalogue. Nevertheless, the generally known great internal error of the photographic photometry does not secure the sufficient accuracy of this method. The corresponding distance is $2.16 \mathrm{kpc}$. Next method (Miroshnichenko 1988) gives the value $E_{B-V}=0.43 \mathrm{mag}$ and the distance of $2.01 \mathrm{kpc}$. We can demonstrate similar scatter of the values of the colour excess also on the basis of papers by Hauschildt et al. (1994, $0.5 \mathrm{mag})$; Elkin (1995, $0.67 \mathrm{mag}$ ) and Lynch et al. (1997, even $1.09 \mathrm{mag})$. Especially the latter two values significantly differ from the ones published by the other authors and also from the values used throughout the present paper. The review of all colour excesses and corresponding distance values is presented in Table 2.

It is also interesting to note the behaviour of the $(B-V)$ index since shortly after the outburst until the dissipation of the dust shell. Immediately after the outburst, the value of the index was virtually constant (the values marked SP in Table 1) and the observed differences were within the measurement error. The index has only begun to change in relation to the formation of the dust shell. But what is especially interesting, its value was still increasing even during the time when the dust shell has already been so rarefied that it virtually did not manifest itself in the visual light curve anymore (Fig. 3). The further observational fact is discernible in the visual light curve at around JD $=2450250$ when there is a brightness decrease by about 1 magnitude. Through this the $(B-V)$ index has probably returned to its original value and the physical influences within the shell deforming previously the overall light curve were definitely off. Although we have no $U B V$ data from this period, we can speculate on the basis of the facts presented earlier here that after further years of the development of the relic shell its extinction influence will be of the order of 0.1 mag above the rough estimate. 
In such a case the presence of the shell is the cause of the underestimate of the distance by $0.4 \mathrm{kpc}$ at most.

The problems with the determination of the correct colour excess of this nova can be caused by the overabundance of the CNO elements. It is probable that novae of the DQ Her subclass, to which V705 Cas unequivocally belongs, characterized by the deep minimum and the dust shell formation, are fundamentally different from the other types of classical novae. As the further support for this, we can point out also the colour index at maximum $(B-V)_{0, \max }=0.05$ mag we have determined for V705 Cas because its value significantly differs from the mean colour index of classical novae at maximum, $0.23 \mathrm{mag}$, derived by van den Bergh \& Younger (1987).

Some results published in this paper have already been presented in preliminary form by one of us (K.P.) during the IAU Coll. No. 157 Cataclysmic Variables and Related Objects in Keele, UK, in 1995 (Petrík et al. 1996). The differences in the derived values of some nova parameters between that preliminary version and the present paper are caused by the use of the different statistical empirical relations.

\section{Conclusions}

Although we share the opinion of Chochol et al. (1997) that since there are well established techniques allowing one to estimate the errors of the absolute magnitude determination of a nova at maximum as well as those of determination of the interstellar extinction (Livio 1992, 1994, 1996; Della Valle \& Livio 1995; Yungelson et al. 1996; Chochol et al. 1993, 1995 and references therein), the analysis based on these methods may not lead to an acceptable nova distance determination for DQ Her stars. Probably the best way how to determine the distance to dusty novae is the use of the nebular expansion parallax method (Chochol et al. 1997).

In future, it would be extremely useful to perform some observational studies of the expanding shell of V705 Cas using high-quality HST data, as in the case of V1974 Cyg (Nova Cygni 1992) earlier.

Acknowledgements. We are indebted to D.K. Lynch who has kindly provided us with a copy of the paper by him and his collaborators prior to publication. We thank the Director of the Hlohovec Observatory for generous allocation of the observational time while the relevant observer (K.P.) was still a student. It is a pleasure to express our sincerest thanks to all the observers communicating their results via the VSNET network for their diligence and patience during the accumulation of the long-term data. Our thanks belong to Michael Friedjung and Massimo Della Valle for their valuable comments while reading the manuscript. This work has been supported in part through the Slovak Academy of Sciences Grant No. 5016/98.

\section{References}

Boyle S.J., 1994, IAU Circ., 6097

Chochol D., Hric L., Urban Z., Komžík R., Grygar J., Papoušek J., 1993, A\&A 277, 103

Chochol D., Hric L., Komžík R., Urban Z., 1995, in Cataclysmic Variables, Abano Terme, Italy, Bianchini A., Della Valle M., Orio M. (eds.). Kluwer, Netherlands, p. 154 Chochol D., Grygar J., Pribulla T., et al., 1997, A\&A 318, 908 Della Valle M., Livio M., 1995, ApJ 452, 704

Elkin V.G., 1995, PisAZh 21, No. 10, 750

Evans A., Geballe T.R., Rawlings J.M., Scott A.D., 1996a, in Cataclysmic Variables and Related Objects, Keele, UK, Evans A. and Wood J.H. (eds.). Kluwer, Netherlands, p. 325

Evans A., Geballe T.R., Rawlings J.M., Scott A.D., 1996b MNRAS 282, 1049

Eyres S.P., Davis R.J., Bode M.F., Lloyd H.M., 1996, IAU Circ., 6292

Gehrz R.D., Greenhouse M.A., Hayward T.L., et al., 1995 ApJ 448, L119

Hauschildt P.H., Starrfield S., Shore S.N., et al., 1994, AJ 108, 1008

Kanatsu K., 1993, IAU Circ., 5902

Kato M., 1994, A\&A 281, L49

Kato M., Hachisu I., 1994, ApJ 437, 802

Livio M., 1992, ApJ 393, 516

Livio M., 1994, in Interacting Binaries, Nussbaumer H. and Orr A. (eds.). Springer-Verlag, Berlin, p. 135

Livio M., 1996, STScI Preprint, No. 1106

Lynch D.K., Russell R.W., Kellogg R.C., et al., 1997, AJ 113, 1391

Miroshnichenko A.C., 1988, AZh. 65, 582

Munari U., Hric L., Tomov T., Hazucha P., 1994a, IBVS 20, No. 3977

Munari U., Tomov T., Antov A., et al., 1994b, IBVS, No. 4005

Petrík K., Hric L., Urban Z., et al., 1996, in Cataclysmic Variables and Related Objects, Keele, UK, Evans A. and Wood J.H. (eds.). Kluwer, Netherlands, p. 323

Pfau W., 1976, A\&A 50, 113

Retter A., Leibowitz E.M., 1995, IAU Circ., 6234

Shore S.N., Starrfield S., Gonzales-Riestra R., et al., 1994a, Nat 369, 539

Shore S.N., Starrfield S., Hauschildt P., 1994b, IAU Circ., 5925

Schmidt B., 1993, IAU Circ., 5905

Schmidt Th., 1957, Zs. Astrophys. 41, 182

Skiff B., 1993, IAU Circ., 5904

Starrfield S., 1988, in Multiwavelength Astrophysics. Cambridge University Press, p. 159

Starrfield S., Hauschildt P., Shore S., et al., 1995, in Cataclysmic Variables, Abano Terme, Italy, Bianchini A., Della Valle M., Orio M. (eds.). Kluwer, Netherlands, p. 267 van den Bergh S., Younger P.F., 1987, A\&A 70, 125

Williams R.E., 1992, AJ 104, 725

Woodward C.E., Greenhouse M.A., 1993, IAU Circ., 5910

Yungelson L., Livio M., Tutukov A., 1996, STScI Preprint, No. 1112 